\title{
Neural systems supporting interoceptive awareness
}

\author{
Hugo D Critchley ${ }^{1-3}$, Stefan Wiens ${ }^{4}$, Pia Rotshtein ${ }^{1}$, Arne Öhman ${ }^{4}$ \& Raymond J Dolan ${ }^{1}$
}

Influential theories of human emotion argue that subjective feeling states-involve representation of bodily responses elicited by emotional events. Within this framework, individual differences in intensity of emotional experience reflect variation in sensitivity to internal bodily responses. We measured regional brain activity by functional magnetic resonance imaging (fMRI) during an interoceptive task wherein subjects judged the timing of their own heartbeats. We observed enhanced activity in insula, somatomotor and cingulate cortices. In right anterior insular/opercular cortex, neural activity predicted subjects' accuracy in the heartbeat detection task. Furthermore, local gray matter volume in the same region correlated with both interoceptive accuracy and subjective ratings of visceral awareness. Indices of negative emotional experience correlated with interoceptive accuracy across subjects. These findings indicate that right anterior insula supports a representation of visceral responses accessible to awareness, providing a substrate for subjective feeling states.

Psychosomatic medicine and psychophysiological science propose an affinity between mental and bodily states ${ }^{1-4}$. Bodily reactions and visceral arousal induce feeling states that color subjective emotional experience ${ }^{5,6}$. The James-Lange theory of emotion defines emotion as perceived central representations of bodily responses to emotive stimuli ${ }^{7,8}$, with emotional feelings (e.g., panic) dependent on bodily responses (e.g. quickening heartbeat) that may be generated automatically by the autonomic nervous system ${ }^{7,8}$. An important prediction arising from these theories is that relative ability to perceive visceral responses will influence measures of subjective affective experience.

Sensitivity to visceral activity can be tested using a heartbeat detection task $k^{1,9-15}$. One class of task involves playing back a signal (visual or auditory), triggered by individual heartbeats, with either a minimal $(<150 \mathrm{~ms})$ or prolonged $(-500 \mathrm{~ms})$ delay $^{9}$. The subject's task is to judge whether feedback is synchronous with their heartbeat. Thus, performance accuracy indexes interoceptive awareness and sensitivity. Subjective emotional experience is reported to relate to degree of interoceptive sensitivity, with independent factors including age, fitness, gender and body fat also influencing heartbeat detection performance ${ }^{1,10-12}$. People classed as 'viscerally aware' are described as more emotionally expressive ${ }^{13}$ and may experience higher-intensity emotions ${ }^{14}$. Moreover, high performance heartbeat detectors (those good at interoceptive tasks) can use interoceptive cues to predict behavioral consequences of subliminal stimuli, consistent with key predictions of the James-Lange theory $y^{7,8,15}$.

Psychological conditions, especially anxiety disorders, are characterized by somatic symptoms and increased attention to autonomic bodily processes ${ }^{16}$. Studies report that individuals with high basal anxiety levels show superior performance on tasks indexing interoceptive sensitivity ${ }^{17-20}$. However, these findings remain equivocal, as many clinical patients with anxiety and panic disorder may perform poorly on tasks such as heartbeat detection while remaining focused on somatic symptoms $s^{21,22}$. This makes a case for distinguishing between neural mechanisms that support attention to interoceptive states and those that support accurate perception of interoceptive information.

Neuroanatomical insights suggest that information concerning the internal state of the body is conveyed through a dedicated lamina-1 spinothalamocortical pathway that converges with vaga afferents, to 'interoceptive centers' in insular and orbitofrontal cortices $^{23}$. Functional neuroimaging suggests a central role for anterior insula cortex (and somatosensory cortex) in mediating subjective feeling states ${ }^{23}$. Anterior insula activity is consistently activated in studies that elicit changes in autonomic arousal ${ }^{24-29}$. It is noteworthy that this region is activated by visceral stimulation ${ }^{30}$, pain $^{31}$, temperature $^{32}$ and emotional processing ${ }^{33,34}$. Right insula cortex activity is also enhanced by awareness of emotionally potent stimuli, suggesting that this region provides an interface between mapping of bodily arousal and representation of these states as subjective feelings ${ }^{26}$

We used fMRI to examine how attention to internal bodily responses and performance accuracy in an interoception task are mapped in the brain. We also rated subjects on questionnaires sensitive to subjective emotional experiences, such as anxiety symptoms, to determine the relationship among subjective feeling states, interoceptive accuracy and brain activity. Furthermore, using voxel-based morphometry ${ }^{35-37}$, we tested for associations between regional differences in brain morphology (gray matter volume) and interoceptive awareness. These convergent approaches suggest that right anterior insula cortex is critical in mediating awareness of interoceptive information contributing to emotional feeling states. 
ARTICLES

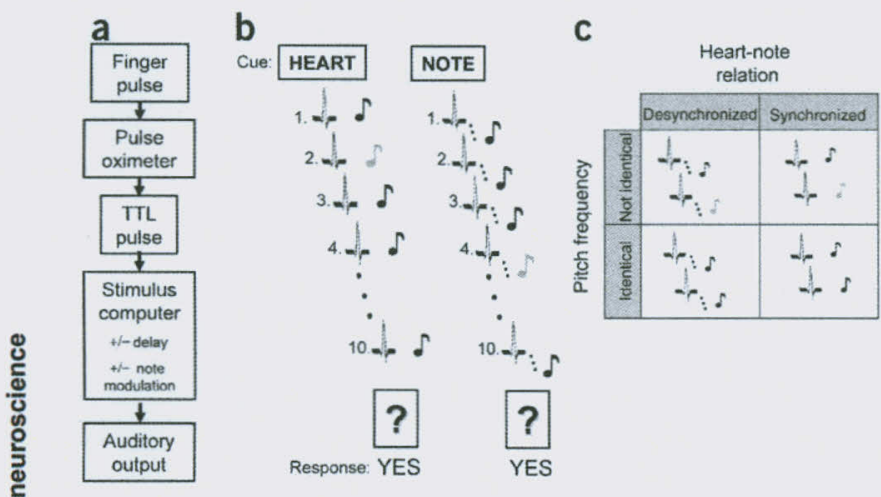

Figure 1 Task design. (a) Experimental setup. The pulse waveform, recorded using pulse oximetry, triggered an auditory feedback note, with or without addition of a 500-ms delay for that trial. Also, on half the trials, one of the ten feedback notes was a different pitch. (b) Eight trial types were presented randomly, reflecting the subjects' attentional focus, the presence or absence of a delay (delay trials are represented here by three dots between pulse waveform and note) and the presence or absence of a modulated note (illustrated in grey). The figure shows two trials. In the first example, the appearance of the word HEART signals trial onset: a subject attends to his own heartbeat and indicates at the end of the trial whether feedback was synchronous or delayed. Ten notes were then played to the subject, triggered by pulses, before the subject was prompted to make the response judgment. The presence or absence of a modulated feedback note was irrelevant to HEART trials. In the adjacent expme a NOTE trial, the subject attends to the quality of the feedback notes, and signals at the end subject attends to the quality of the feedback notes, and signals at the end
whether the notes were all the same (yes) or if one was different (no). The presence or absence of feedback delay was irrelevant to NOTE trials. (c) The study represented a $2 \times 2 \times 2$ factorial design, the principal factors being attention to heartbeat or note quality (cued at trial onset), and presence or absence of a modulated note and of feedback delay. The modulation of feedback timing and note is illustrated in the box.

\section{RESULTS}

Heartbeat detection, interoceptive awareness and emotion

Seventeen subjects were scanned while performing an interoceptive heartbeat detection task (in which they judged the timing of their own heartbeat relative to feedback notes), interleaved with trials of an exteroceptive control task (judging only note quality). The study involved a fully balanced factorial design (see Methods and Fig. 1).

Judgment accuracy in heartbeat detection varied between chance $(46 \%$ ) and $83 \%$ (mean $62 \%$, s.d. $9 \%$ ). At debriefing, all subjects reported that they shifted attention appropriately to their heartbeat timing during interoceptive HEART trials and to note quality during exteroceptive NOTE trials. Two subjects reported guessing during interoceptive trials, whereas four subjects were confident that they performed the interoceptive task accurately. Remaining subjects felt they performed above chance and, overall, estimated performance reflected achieved scores. Performance on the exteroceptive task (NOTE trials) matched that of the interoceptive heartbeat detection task in subjective difficulty and performance accuracy (mean $68 \%$, range $50-88 \%$, s.d. $11 \%$ ). The trend for subjects to be more successful on the NOTE relative to HEART trials did not reach significance $\left(t_{32}=1.8, P=0.07\right)$.

Thirteen subjects returned clinical questionnaires indexing symptoms of anxiety ${ }^{38}$, depression ${ }^{39}$ and positive and negative affective experience (PANAS score ${ }^{40}$; see Methods). All scored below diagnostic criteria for anxiety and depressive disorder. Across subjects, anxiety score correlated with interoceptive accuracy during scanning (relative to exteroceptive accuracy, to control for non-specific performance effects during scanning; Pearson $R=0.64, P<0.05$ ). Relative interoceptive accuracy tended to correlate with depressive symptoms $(R=0.48, P=0.09)$ and trait ratings of negative affective experience $(R=0.51, P=0.08)$. Trends in correlation between interoceptive accuracy and negative emotions remained even when nonspecific performance effects during scanning were ignored (number of correct HEART trials and anxiety score, $R=0.513, P=0.075$; correct HEART trials and depression score, $R=0.481, P=0.096)$. Anxiety symptoms correlated strongly with both depression $(R=0.82, P<0.01)$ and negative affect (trait) scores $(R=0.76, P<0.01)$. No correlations were observed between heartbeat detection and 'state' PANAS score or scores of positive emotional experience $(R=-0.01, P=0.76)$.

Twenty-five subjects, who participated in a morphometric study, completed a body perception questionnaire ${ }^{41}$, which consisted of five subtests relating to stress and perception of bodily responses. Scores were consistent with normative data, with subjects scoring slightly below average on the "awareness of bodily processes" subtest (mean \pm s.d, $2.5 \pm 0.5$, normative score $3.0 \pm 0.8)^{41}$. Interestingly, we found no correlation between heartbeat detection accuracy and subtests of the body perception questionnaire, including the awareness score.

\section{Functional imaging data}

Neuroimaging data was analyzed to determine significant group effects (see Methods). Interoceptive (heartbeat detection), compared to exteroceptive attention (note detection), was associated with enhanced activity in bilateral regions of lateral somatomotor cortex, anterior insula and inferior frontal cortex. Activity in dorsal anterior cingulate cortex and supplementary motor cortex was also enhanced during interoceptive attention $(P<0.02$ corrected; Table 1a and Fig. 2a). In contrast, regions of occipital cortex showed decreased activity during attention to heartbeat.

Perceptual matching of feedback to interoceptive responses is critical for accurate performance of the heartbeat detection task. We observed no significant main effect of feedback delay. Nevertheless, activity in frontal operculum and insula, dorsal and medial parieta lobe, right dorsolateral prefrontal cortex, dorsal cingulate and latera temporal cortices reflected an interaction between feedback delay and attentional focus ( $P<0.02$, corrected; Table 2 and Fig. $2 b)$. Activity in these regions showed a greater difference between delay trials and synchronous trials during interoceptive attention. Using a masking

Table 1 Activity related to interoception and feedback delay

\begin{tabular}{|c|c|c|c|}
\hline \multicolumn{4}{|c|}{ a Enhanced activity during heartbeat detection } \\
\hline Location & Side & Coordinates & $t$-value \\
\hline $\begin{array}{l}\text { Inferior parietal lobule/ } \\
\text { post central gyrus }\end{array}$ & L & $-58,-32,38$ & 5.71 \\
\hline \multicolumn{4}{|l|}{ Lateral somatomotor cortex } \\
\hline & $\mathrm{R}$ & $54,-22,28$ & 5.00 \\
\hline & $\mathrm{R}$ & $60,-18,32$ & 4.83 \\
\hline Insula/operculum & $\mathrm{R}$ & $32,16,10$ & 5.52 \\
\hline \multirow[t]{3}{*}{ Insula cortex } & $\mathrm{R}$ & $62,18,6$ & 4.70 \\
\hline & L & $-44,12,-4$ & 4.54 \\
\hline & $\mathrm{R}$ & $56,14,4$ & 4.66 \\
\hline Inferior frontal gyrus & L & $-48,32,4$ & 4.73 \\
\hline Inferior frontal gyrus & $\mathrm{R}$ & $54,44,2$ & 4.07 \\
\hline Supplementary motor area & L & $-10,-18,70$ & 4.63 \\
\hline Anterior cingulate & $\mathrm{R}$ & $6,-2,48$ & 4.13 \\
\hline \multicolumn{4}{|c|}{ b Decreased activity during heartbeat detection } \\
\hline Location & Side & Coordinates & $t$-value \\
\hline Striate/extrastriate cortices & $\mathrm{L}$ & $-16,-66,2$ & 4.64 \\
\hline
\end{tabular}


Figure 2 Activity relating to interoceptive attention (second-level random effects analysis of 17 subjects, $P<0.02$ corrected). (a) Main effect of interoceptive attention. Regional enhancement of brain activity during HEART trials, requiring interoceptive attention, compared to control NOTE trials. Group activity is plotted on horizontal sections of a normalized is ploted on horizol template bran to illystrate activation in bilater anterior insula (Ins), lateral somatomotor and adjacent parietal cortices $(\mathrm{Sm})$, anterior cingulate (ACC) and supplementary motor cortices (SMA). Also indicated are right (R) and left (L), and height $(\mathrm{mm})$ of each of axial slice. (b) Activity reflecting interaction between feedback delay relative to heartbeat and interoceptive focus. Group activity is plotted on orthogonal sections of a template image to illustrate opercular (FO), anterior cingulate (ACC), medial parietal (MP) and thalamic activity (th) associated with contextual processing of feedback relative to interoceptive information $(P<0.02$, corrected). Left $(L)$ is indicated on coronal and axial sections. (c) Glass brain projection of activity identified in group analyses of both the main effect of interoceptive

attention, and in interaction between interoceptive attention and feedback delay. An inclusive mask of the main effect $(P<0.02$, corrected was used to constrain analysis of the interaction. The peak conjoint activity in right anterior insula/opercular cortex is marked, and the parameter estimates (with $90 \%$ confidence intervals) plotted. In this figure, and subsequent plots of neuorimaging data, units are given in arbitrary units adjusted for confounding effects. For fMRI data, units are proportional to percentage signal change. Interoceptive effects are represented by the bars on the left, with synchronous trial effects in blue and delayed trial effects in orange. b

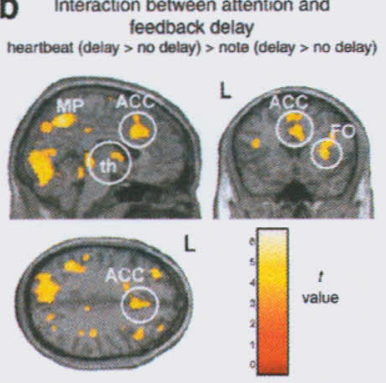

C Interaction masked inclusively by main

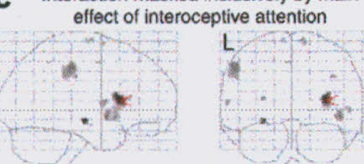

Anterior insula / operculum
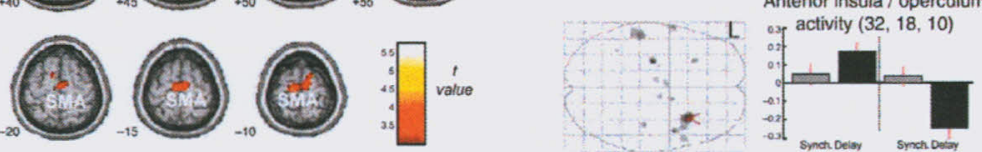

procedure, we tested whether the same region of right anterior insular/opercular cortex expressed both the main effect of interoceptive attention and interaction between interoception and feedback. We found that activity here was driven primarily by differences during delay trials between interoceptive versus exteroceptive attention, reflecting mismatch between heartbeat and feedback when attention was focused internally. The same feedback mismatch suppressed right anterior insula activity when attention was externally directed to tones, indicating that activity in this region is modulated as a function of interoceptive or exteroceptive attention (Fig. 2c).

Having highlighted regions modulated by interoceptive attention, we tested for activity mediating interoceptive awareness, correlating with performance of the heartbeat detection task. We undertook a group analysis that modeled performance accuracy on heartbeat and note detection tasks as, respectively, a regressor-of-interest and confounding covariate (see Methods). Within regions where activity was enhanced by interoceptive attention, activity in right anterior insular/opercular cortex showed the strongest relationship with interoceptive accuracy $(34,20,4 ; t=3.09, P<0.02$ corrected for small volume; Fig. 3a). Activity within this region during heartbeat detection trials correlated strongly with interoceptive accuracy (relative to exteroceptive accuracy) $(R=0.62$, Fig. $3 \mathrm{~b})$

The theoretical relationship between interoceptive awareness and emotional feeling states was reinforced by the observation that subjective anxiety symptoms ${ }^{38}$, correlating with relative interoceptive accuracy (Fig. 3c), also correlated with mean activity in right anterior insular/opercular cortex $(R=0.65, P<0.05)$ during heartbeat detection trials (Fig. 3d). Together, these functional imaging findings highlight anterior insula cortex, somatomotor cortex and anterior cingulate in supporting interoceptive attention, with right anterior insular/opercular activity reflecting interoceptive sensitivity, explicit awareness of bodily processes and emotional feeling states.

\section{Morphometric analyses}

We next used voxel-based morphometry (see Methods) to test for a relationship between brain structure (regional gray matter volume) and interoceptive awareness (relative accuracy on the heartbeat detection task). Detailed structural brain images were obtained for 15 of 17 participants in the functional imaging task. We found that gray matter volume in right anterior insula/operculum $(33,28,13, t=11.8)$ orbitofrontal cortex and midline cerebellum correlated with relative interoceptive accuracy (Fig. 4a,b).

In an extended group of healthy individuals $(n=25)$, we tested the relationship between regional brain morphometry and scores on the awareness subtest score of the body perception questionnaire ${ }^{41}$. This subtest requires subjects to rate awareness of visceral responses on a

Table 2 Activity reflecting interaction between attention to heartbeat and feedback delay

\begin{tabular}{|c|c|c|c|}
\hline \multicolumn{4}{|c|}{ Heartbeat (delay $>$ no delay) $>$ Note (delay $>$ no delay) } \\
\hline Location & Side & Coordinates & $t$-value \\
\hline Medial parietal lobe/precuneus & $\mathrm{R}$ & $10,-60,46$ & 6.55 \\
\hline \multicolumn{4}{|l|}{ Superior parietal lobule } \\
\hline & $\mathrm{R}$ & $26,-50,58$ & 4.97 \\
\hline & L & $-36,-64,42$ & 4.44 \\
\hline Anterior insula/frontal operculum & $\mathrm{R}$ & $38,18,10$ & 5.05 \\
\hline Posterior insula & $\mathrm{R}$ & $48,2,-6$ & 4.41 \\
\hline \multirow[t]{2}{*}{ Dorsolateral prefrontal cortex } & L & $-36,40,36$ & 4.59 \\
\hline & $\mathrm{R}$ & $36,26,26$ & 4.33 \\
\hline Inferior frontal gyrus & L & $-40,22,20$ & 4.58 \\
\hline Occipital cortices & $\mathrm{R}$ & $30,-80,22$ & 4.85 \\
\hline Middle temporal gyrus & L & $-56,-48,0$ & 4.82 \\
\hline Superior temporal sulcus & $\mathrm{R}$ & $58,-30,0$ & 4.67 \\
\hline Dorsal anterior cingulate & - & $6,18,34$ & 4.23 \\
\hline
\end{tabular}



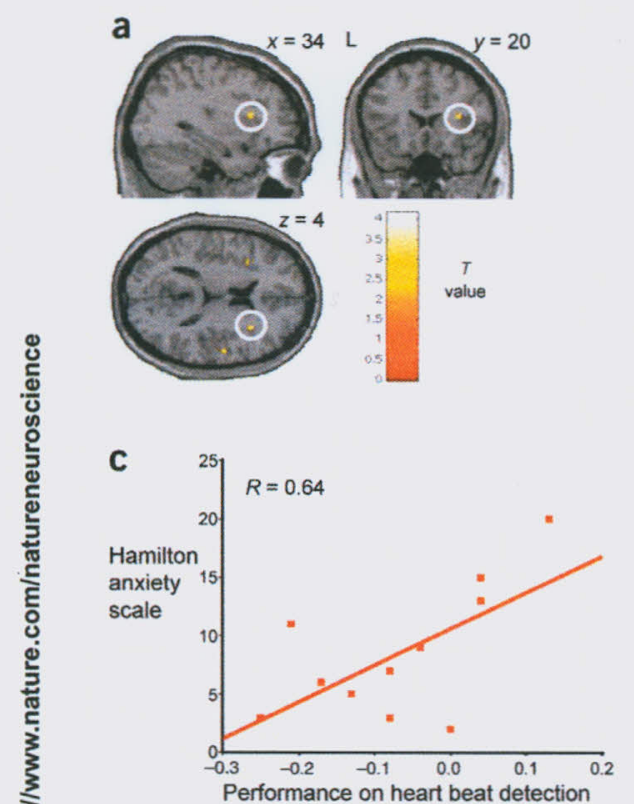

task (relative to note task)

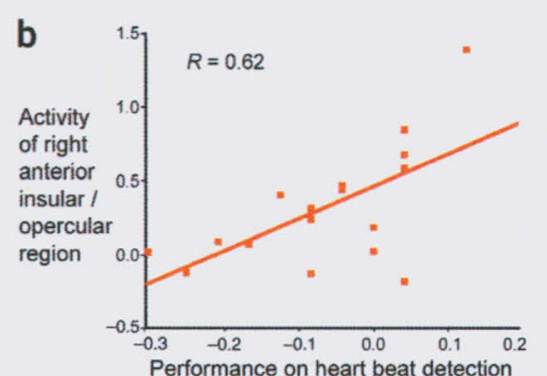

task (relative to note task)

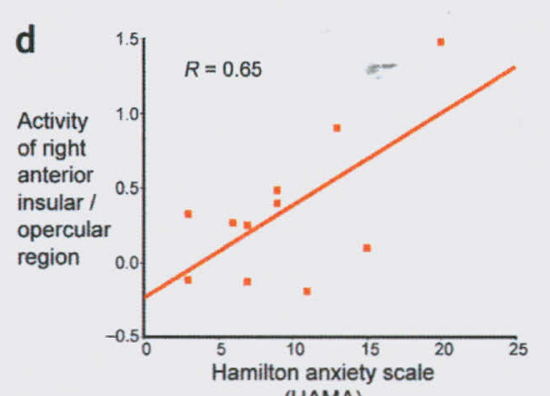

(HAMA)
Figure 3 Functional neural correlates of interoceptive sensitivity. (a) Activity in right anterior insula/opercular activity correlated with performance accuracy on the heartbeat detection task in an analysis that modelled both interoceptive and exteroceptive task performance separately. The anatomical location is mapped on orthogonal sections of a template brain, with coordinates in $\mathrm{mm}$ form anterior commissure. (b) Activity within right insular/opercular cortex during interoceptive trials is plotted against interoceptive accuracy (relative to exteroceptive accuracy, to control for non-specific detection difficulty in the noisy scanning environment). The Pearson correlation coefficient $(R)$ is given in the plot. (c) Subject scores on the Hamilton Anxiety Scale $\left(\mathrm{HAMA}^{38}\right)$ are plotted against relative interoceptive awareness to illustrate the correlation in these subjects between sensitivity to bodily responses and subjective emotional experience, particularly of negative emotions. (d) Activity in right anterior insula/opercular activity during interoception also correlated with anxiety score ${ }^{38}$, suggesting emotional feelings states are supported by explicit interoceptive representations within right insula cortex. five-point scale. Questions begin with "During most situations I am aware of:" and exemplars include "swallowing frequently," "eye fatigue," and "stomach and gut pains". To test for specific gray matter associations, we modeled the awareness scores as a regressor-of-interest, and observed a positive correlation $(R=0.35, P<0.01)$ with gray matter volume in right frontal operculum/anterior insular $(45,28,8$, $t=4.77$; Fig. 5)

\section{DISCUSSION}

Using functional neuroimaging, we identified a matrix of cortical regions supporting interoceptive attention involving right oper cular, bilateral insular, somatomotor, parietal cortices and supplementary motor/anterior cingulate cortices. The central role of somatosensory and insula cortices in interoceptive attention endorses their proposed contribution to subjective feeling states arising from representations of bodily responses ${ }^{23,42,43}$. Regions integrating intero-

Figure 4 Morphometric (gray matter) correlates of interoceptive sensitivity. (a, b) Voxel-based morphometry was used to determine the relationship between regional gray matter volume and interoceptive sensitivity (relative to heart beat detection accuracy), highlighting effects in (a) right anterior insula operculum and (b) right orbitofrontal cortex. Medial cerebellar gray matter volume also reached threshold

matter volume also reached threshold
significance. Group data is plotted on orthogonal sections of a normalized template brain (with coordinates in $\mathrm{mm}$ from anterior commissure). Adjacent, the adjusted measure of regional gray matter volume for each region is plotted against interoceptive performance. Pearson correlation coefficients $(R)$ are also given. ceptive information, including anterior cingulate, ventromedial prefrontal and lateral prefrontal cortices, were additionally recruited during processing of feedback timing relative to heartbeat and may represent a substrate for a contextual "second-order representation of self" 25,43 .

Our fMRI findings of enhanced right anterior insular/opercular cortex during interoceptive attention endorses two previous proposals $^{23,26}$ that this region mediates interoceptive awareness. Activity in this region correlated with interoceptive accuracy, reflecting explicit

Increased gray matter with increased heart beat detection accuracy
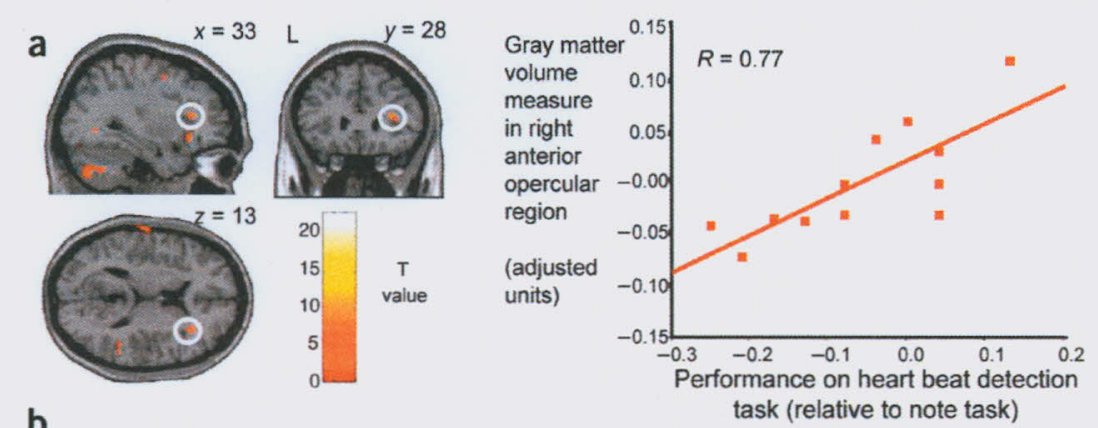
task (relative to note task)
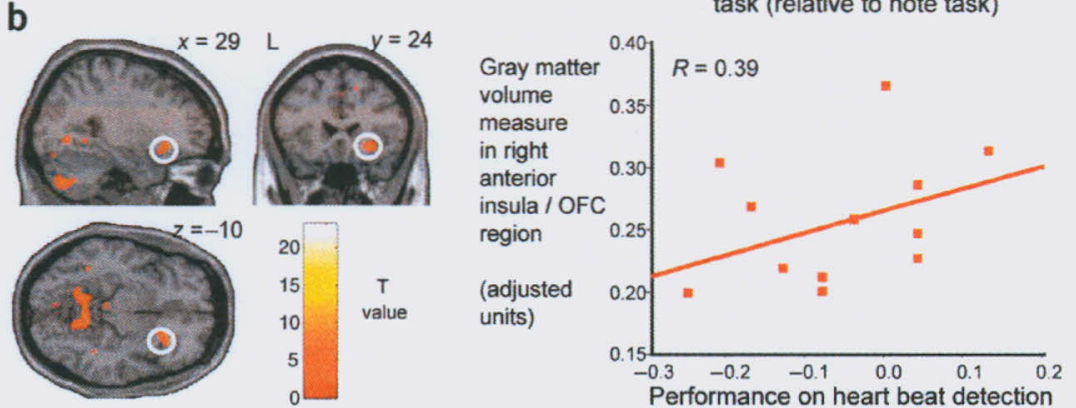

task (relative to note task) 
awareness of bodily processes, and importantly, with measures of subjective emotional experience. Moreover, gray matter volume within right anterior insula/operculum predicted objective measures of interoceptive performance during scanning, and subjective rating of general visceral awareness. We propose that representation of internal bodily states instantiated by anterior insular/opercular cortex is available to conscious awareness as subjective feeling states.

Insula cortex, usually in concert with anterior cingulate, is activated during states of autonomic arousal ${ }^{24-32,34}$. However, in contrast to cingulate regions implicated in generating contextual arousal states ${ }^{27}$, insula cortex may support afferent representation of bodily states, as suggested by relative deactivation when autonomic arousal is absent during behavioral or emotional challenge ${ }^{25,26}$. Whereas interoceptive attention enhanced activity in a number of cortical regions receiving afferent information about bodily states, only activity in right anterior insula reflected explicit awareness of interoceptive information. Moreover, we identified this region as a substrate for interoceptive awareness without stimulus confounds or changes in arousal level that are integral to studies mapping responses elicited by thermal or visceral stimulation ${ }^{24-32}$.

Enhanced somatic symptoms and attention to interoceptive states are characteristic of anxiety disorders ${ }^{16}$. The observed interrelationship among right anterior insula activity, interoceptive accuracy and subjective negative emotional experience supports the proposal that affective feeling states reflect information concerning bodily responses represented in right anterior insula ${ }^{42,43}$. In contrast with earlier findings ${ }^{14}$, we observed no relationship between interoceptive accuracy and experience of positive emotions. Nevertheless, our observation of an association with anxiety symptoms has previously received greater emphasis ${ }^{17-22}$. The proposal that right anterior insula mediates somatic and visceral attention is further strengthened by correlations between local gray matter volume and questionnaire ratings of somatic awareness ${ }^{41}$. Intriguingly, despite convergence of our morphometric and functional imaging findings, we did not find a relationship between self-rated bodily awareness and performance accuracy on heartbeat detection. Nevertheless, both data sets implicate right anterior insular/opercular cortex in supporting awareness of visceral responses that are enhanced in negative mood states.

It has been argued ${ }^{43}$ that core consciousness and sense of 'self' are grounded in representations of homoeostatic bodily responses in the context of ongoing behavior. Subjective feeling states and emotions emanate from representations of these somatic processes. A firstorder representation of self is proposed within brainstem autoregulatory centers, primary and secondary somatosensory cortices and insula ${ }^{25,43}$. Second-order 'meta' representations are proposed to be supported within ventromedial prefrontal and cingulate cortices. Indeed, empirical evidence implicates anterior cingulate in secondorder remapping of autonomic responses ${ }^{25}$. A departure from the previous model ${ }^{43}$ arises from our observation that right anterior insula cortex may integrate interoceptive and external information, apparent in the interaction between attentional focus and feedback delay, and in an earlier study ${ }^{26}$. This suggests a comparator role for anterior insula that extends beyond first-order representation of interoceptive states, yet qualitatively different from the type of dynamic second-order representation proposed for cingulate cortex ${ }^{43}$.

It has also been proposed that the role of right anterior insula in explicit representation of feeling states may represent an evolutionary specialization unique to primates, consequent upon development of lamina-1 thalamocortical neural pathways that convey detailed interoceptive information ${ }^{23}$. Insula projections of this thalamocortical system support convergence of bodily information including meta-
Gray matter volume correlates of 'awareness' subtest of the Body Perception Questionnaire ${ }^{41}$
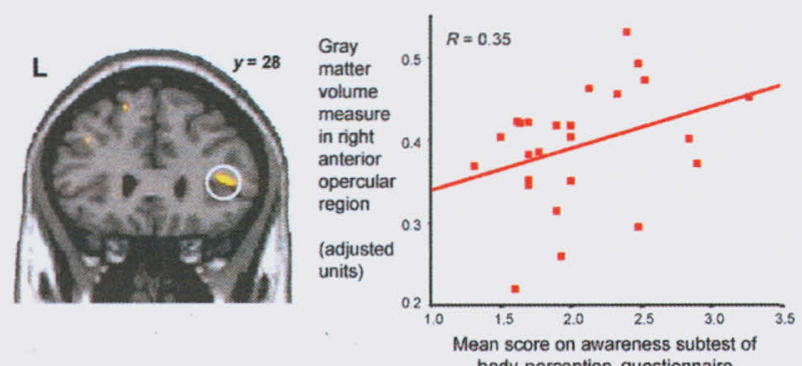

body perception questionnaire

Figure 5 Gray matter correlates of measures of self-rated bodily awareness. Regional gray matter volume in right anterior insula/operculum correlated in 25 subjects with score on the awareness subtest of a body perception questionnaire $e^{41}$, plotted at $P<0.05$, corrected on a coronal section of a template brain image and adjacent as a scatterplot of awareness score and adjusted data for local gray matter volume. The Pearson correlation $(R)$ is given in the scatterplot frame.

boreception, pain, itch, temperature and visceral changes to provide a rich mapping of internal states for representations of self. Interestingly, parallel specialization of human dorsal cingulate cortex is proposed for expression of motivational drives, wherein interoceptive information is conveyed via an ancillary medial lamina-1 thalamocortical pathway relaying in ventrocaudal sectors of mediodorsal thalamus ${ }^{23}$. This anatomical arrangement accounts for co-activation of anterior cingulate and insula cortices by interoceptive processes, including cardiovascular arousal ${ }^{27}$ and visceral stimulation ${ }^{30}$, despite dissociation of efferent, and afferent autoregulatory, functions ${ }^{25-27}$.

In summary, we used functional and structural neuroimaging techniques to identify brain regions that contribute to attention and awareness of internal bodily processes. Our findings suggest that insula mediates attention to, and right anterior insula/operculum mediates explicit awareness of, internal bodily processes. Moreover, the relationship among interoceptive awareness, subjective emotional experience and right anterior insula activity suggests a neuroanatomical substrate for feeling states that may underlie a conscious representation of self.

\section{METHODS}

Subjects and questionnaires. These neuroimaging studies were approved by the Joint Ethics Committee of the Institute of Neurology and National Hospital for Neurology and Neurosurgery. Subjects gave informed written consent. Seventeen healthy subjects ( 9 female, 8 male), recruited for the functional imaging study, completed clinical questionnaires indexing symptoms of anxiety (Hamilton anxiety scale, HAMA $^{38}$ ), depression (Beck Depression Inventory, $\mathrm{BDI}^{39}$ ) and positive and negative affective experience (PANAS ${ }^{40}$ scored twice to quantify state ('right now, at the present moment') and trait (generally, how you feel on average) experiences of positive and negative feelings). Thirteen subjects returned these questionnaires, which were used in correlational analyses with heartbeat detection performance and regional brain activity. Additionally, twenty-five subjects underwent structural T1 MRI scans on the same scanner and completed the body perception questionnaire ${ }^{41}$, a five part self-rated questionnaire probing awareness of bodily and stress responses. The awareness dimension of this questionnaire was used as a regressor-of-interest in a voxel-based morphometry analysis.

Functional imaging study of heartbeat detection. Subjects underwent MRI scanning at 1.5 tesla (Siemens Sonata). Mild external head restraint was used to minimize head movement during scanning. Whole-brain data were 


\section{ARTICLES}

acquired using echoplanar imaging, sensitive to BOLD contrast (28 slices, $3.5 \mathrm{~mm}$ thick, tilted $-30^{\circ}$ from intracommisural plane, $\mathrm{TE}=40 \mathrm{~ms}, \mathrm{TR}=2.52$ $s$ per volume). Heart rate was monitored using a pulse oximeter (Nonin8600, Nonin Medical, Inc.); the pulse probe was placed on the left middle finger. Onset of each pulse waveform triggered an auditory feedback note in the experimental task. Subjects were presented with visual cues via a projector screen, and auditory feedback via headphones. A button box enabled recording of responses.

Experimental task. During functional scanning, subjects performed repeated randomized trials of a task that required them to attend to either their heartbeat timing or external note quality (Fig. 1). Each trial began with visual presentation of either the word HEART or NOTE. The subject then heard a sequence of ten notes ( $100 \mathrm{~ms}$ duration), each note triggered by onset of the subject's pulse. In half of the trials, auditory feedback was delivered immediately. In the other half, each note was delivered after a 500 -ms delay period. Moreover, in half the trials, one note (randomized) was subtly different in pitch to the other nine notes (a pure tone at $800 \mathrm{~Hz}$ was changed to $785 \mathrm{~Hz}$ ). At the end of each trial, the subject made a forced-choice button-press response, prompted by visual presentation of a question mark. For HEART trials, one response indicated that the subject perceived no feedback delay, and another indicated a perceived delay. For NOTE trials, one response indicated that the subject perceived no difference in pitch among the notes, and another that a difference was perceived. Thus, there were eight trial types, randomized over 56 trials, reflecting permutations of attention to HEART or NOTE, presence or absence of feedback delay, and presence or absence of a rogue feedback note. The total duration of the experiment reflected the subject's resting heart rate, but was consistently less than $15 \mathrm{~min}$.

Analyses of functional data set. The fMRI time series data were analyzed using SPM2 (http://www.fil.ion.ucl.ac.uk/spm/spm2.html) on a Matlab platform SPM2 (http://www.fil.ion.ucl.ac.uk/spm/spm2.html) on a Matlab platform
(Mathworks Inc.). Echoplanar images were corrected for slice timing and head (Mathworks Inc.). Echoplanar images were corrected for slice timing and head subject, an analytic design matrix was constructed, modeling onsets and duration of each trial as epochs convolved with a hemodynamic response function. The eight trial types were modeled as separate regressors and interrogated to The eight trial types were modeled as separate regressors and interrogated to
derive contrast images for second-level (group) ANOVA and correlational analyses using SPM $2^{44-47}$. Contrasts for presence and absence of feedback delay for heartbeat and note detection conditions were entered into a group ANOVA that explored main effects and interaction of interoceptive attention and feedback delay. Next, we undertook a regression analysis of contrast images for HEART versus NOTE detection trials that modeled interoceptive and exteroceptive performance accuracy separately as covariates. Threshold significance for functional imaging data was $P<0.02$, corrected using false discovery rate (FDR) correction for multiple comparisons ${ }^{47}$. A mask image generated from the main effect of interoceptive attention $(P<0.02$, corrected $)$ was used for small-volume region-of-interest correction of performance-related activity (Fig. 3a).

Structural volumetric correlates of interoceptive awareness. We used optimized voxel-based morphometry ${ }^{35-37}$ to explore associations between regional gray matter volume and indices of interoceptive awareness. T1weighted whole-brain structural images were obtained using a 3D-spoiled FLASH gradient echo sequence, field of view $224 \times 256 \mathrm{~mm}, 1 \mathrm{~mm}$ slice thickness, $T R=16.0 \mathrm{~ms}, \mathrm{TE}=9.0 \mathrm{~ms}$ ). First, in 15 subjects, we examined how interoceptive performance (during functional imaging) related to regional gray matter volume. Next, in 25 subjects, we tested for correlations between regional gray matter volume and scores on the awareness subtest of the body perception questionnaire ${ }^{41}$.

Morphometric data analyses used an optimized voxel-based morphometry (VBM) protocol in SPM. Automated image pre-processing steps (brain extraction, spatial normalization, segmentation, modulation and smoothing $)^{35-37}$ produced normalized, modulated gray matter images for each subject with reference to a group template image. Modulation corrects for volume changes introduced during non-linear spatial transformations and enables analysis of associations with absolute amount (volume) of regional gray matter. Images were smoothed with a 12 -mm full-width-half-maximum isotropic Gaussian kernel to conform residuals to the Gaussian random field model underpinning statistical inferences ${ }^{44-47}$. Regional relationships between gray matter volume and indices of interoceptive awareness (relative performance on the heartbea detection task) and score on the awareness subtest of the body perception questionnaire, were assessed in separate analyses using global gray matter signal, age and gender as confounding covariates. Morphometric effects were reported when significance reached $P<0.05$, corrected (FDR) for whole brain or small volume-of-interest (insular cortex).

\section{ACKNOWLEDGMENTS}

This work was supported by a Wellcome Clinician Scientist Fellowship to H.D.C. and a Programme Grant to R.J.D.

\section{COMPETING INTERESTS STATEMENT}

The authors declare that they have no competing financial interests.

Received 29 October; accepted 26 November 2003

Published online at $\mathrm{http}: / / \mathrm{ww}$.nature.com/natureneuroscience

Cameron, O.G. Interoception: the inside story-a model for psychosomatic

proces. P. Mind

. action. Psychol. Res. 66, 337-349(2002).

4. Jennings, J.R. Is it important that the mind is in a body? Inhibition and the heart. Psychophysiology 29, 369-383 (1992).

Damasio, A.R. Descartes' Error: Emotion, Reason and the Human Brain (Grosset/Putnam, New York, 1994).

. Dolan, R.J. Emotion, cognition, and behavior. Science 298, 1191-1194 (2002)

. James, W. Physical basis of emotion. Psychol. Rev. 1. 516-529 (1894). Reprinted in Psychol. Rev. 101, 205-210 (1994).

Lange, C. The Emotions (Williams \& Wilkins, Baltimore, Maryland, 1922 Translated by I.A. Haupt; original work published 1885 .

Whitehead, W.E., Drescher, V.M., Heiman, P. \& Blackwell, B. Relation of heart rate control to heart beat perception. Biofeedback and Self Regulation 2, 371-392 (1977)

0. Yates, A.J., Jones, K.E., Marie, G.V. \& Hogben, J.H. Detection of the heart beat and events in the cardiac cycle. Psychophysiology 22, 561-567 (1985).

Wiens, S. \& Palmer, S.N. Quadratic trend analysis and heart beat detection. Biol. Psychol. 58, 159-175 (2001).

, E. Perception of visceral sensations: a review of recent findings, methodologies and future directions. in Advances in Psychophysiology Vol. 5 (eds. (essica Kingsley, London, 1994). Psychol. $42,131-145$ (1996).

4. Wiens, S., Mezzacappa, E. \& Katkin, E.S. Heart beat detection and the experience of emotion. Cognit. Emotion 14, 417-427 (2000)

5. Katkin, E.S., Wiens, S. \& Ohman, A. Nonconscious fear conditioning, visceral perception, and the development of gut feelings. Psychol. Sci. 12, 366-370 (2001) 6. Mumford, D.B. et al. The Bradford Somatic Inventory. A multi-ethnic inventory of somatic symptoms reported by anxious and depressed patients in Britain and the Indo-Pakistan subcontinent. Br. J. Psychiatry 158, 379-386 (1991).

Tudwick-Rosenthal, R. \& Neufeld, R.W. Heart beat interoception: a study of individ ual differences. Int. J. Psychophysiol. 3, 57-65 (1985).

. Ehlers, A. \& Breuer, P. How good are patients with panic disorder at perceeving their heart beats? Biol. Psychol. 42, 165-182 (1996). 37, 141-158 (1999). . rate reactivity in ancie

Inhy, A.J. Palpitations, arrhythmias, and awareness of cardiac activity. Ann Intern. Med. 134, 832-837 (2001).

Van der Does, A.J.W., Antony, M.M., Ehlers, A. \& Barsky, A.J. Heart beat perception in panic disorder: a

Craig, A.D. How do you feel? Interoception: the sense of the physiological condition of the body. Nat. Rev. Neurosci. 3, 655-666 (2002).

4. Critchley, H.D., Corfield, D.R., Chandler, M. Mathias, C J. \& Dolan, R. Cerebra correlates of peripheral cardiovascular arousal: a functional neuroimaging study. J. Physiol. 523, 259-270 (2000).

5. Critchley, H.D., Mathias, C.J. \& Dolan, R.J. Neural correlates of first and secondorder representation of bodily states. Nat. Neurosci. 4, 207-212 (2001).

. Critchley, H.D., Mathias, C.J. \& Dolan, R.J. Fear-conditioning in humans: the influence of awareness and arousal on functional neuroanatomy. Neuron 33, 653-663 (2002)

27. Critchley, H.D. et al. Human cingulate cortex and autonomic cardiovascular contro converging neuroimaging and clinical evidence. Brain 216, 2139-2152 (2003). 8. Cameron, O.G. \& Minoshima, S. Regional brain activation due to pharmacologically induced adrenergic interoceptive stimulation in humans. Psychosom. Med. 64 
29. Harper, R.M., Bandler, R., Spriggs, D. \& Alger, J.R. Lateralized and widespread brain activation during transient blood pressure elevation revealed by magnetic resonance imaging. J. Comp. Neurol. 417, 195-204 (2000).

30. Aziz, Q., Schnitzler, A. \& Enck, P. Functional neuroimaging of visceral sensation. J. Clin. Neurophysiol. 17, 604-612 (2000).

31. Peyron, R. et al. Role of operculoinsular cortices in human pain processing: converging evidence from PET, fMRI, dipole modeling, and intracerebral recordings of evoked potentials. Neurolmage 17, 1336-1346 (2002).

32. Craig, A.D., Chen, K., Bandy, D. \& Reiman, E.M. Thermosensory activation of insular cortex. Nat. Neurosci. 3, 184-190 (2000).

33. Phillips, M.L. et al. Neural responses to facial and vocal expressions of fear and disgust. Proc. R. Soc. Lond. B Biol. Sci. 265, 1809-1817 (1998).

34. Buchel, C., Morris, J., Dolan, R.J. \& Friston, K.J. Brain systems mediating aversive

35. Good, C.D., et al. A voxel-based morphometric study of ageing in 465 normal adult human brains. Neuroimage 14, 21-36 (2001).

(the methods. Neuroimage

Friston, KJ. Why voxel-based morphometry should be used. Neuroimage 14,1238-1243(2001).
38. Hamilton, M.C. Hamilton anxiety scale [HAMA]. in Sourcebook of Adult Assessment: Applied Clinical Psychology (eds. Schutte, N.S. \& Malouff, J.M. 154-157 (Plenum Press, New York, 1995).

39. Beck, A.T. \& Steer, R.A. Manual for the Beck Depression Inventory (The Psychological Corporation, San Antonio, Texas, USA 1993).

.Watson, $D$. Intra-individual and inter-individual analyses of pos the affect: their relation to health complaints, perceived stress, and daily activities. J. Personality Soc. Psychol. 54,1020-1030 (1988).

1. Porges, S. Body Perception Questionnaire (Laboratory of Developmental Assessment, University of Maryland, 1993).

New York, 1994).

3. Damasio, A.R. The Feeling of What Happens: Body and Emotion in the Making of Consciousness (Harcourt Brace, New York, 1999).

4. Friston, K. et al. Statistical parametric maps in functional imaging: a general linear approach. Human Brain Mapp. 2, 189-210 (1995).

Friston, K. et al. Spatial registration and normalization of images. Human Brain Mapp. 2, 165-189 (1995).

46. Ashburner, J., Neelin, P., Collins, D.L., Evans, A. \& Friston, K. Incorporating prior knowledge into image registration. Neuroimage 6, 344-352 (1997).

. Genovese, C.R., Lanar NA. \& Nichols, T. Thesholding or statsical maps in functiona 